\title{
Exclusive production of two and four pions in ultraperipheral, ultrarelativistic collisions
}

\author{
Mariola KŁUSEK-GAWENDA ${ }^{* a}$ and Antoni SZCZUREK ${ }^{a b} \dagger$ \\ ${ }^{a}$ The Henryk Niewodniczański Institute of Nuclear Physics PAN, PL-31-342 Kraków \\ ${ }^{b}$ University of Rzeszów, PL-35-959 Rzeszów \\ E-mail: Mariola.Klusek@ifj.edu.pl, Antoni.Szczurekeifj.edu.pl
}

\begin{abstract}
The cross section for $\pi^{+} \pi^{-}$and $\pi^{0} \pi^{0}$ meson pairs production in peripheral ultrarelativistic heavy-ion collisions is calculated in the impact parameter space equivalent photon approximation at the energy available at the CERN Large Hadron Collider, $\sqrt{s_{N N}}=3.5 \mathrm{TeV}$. For the first time the world data for $\gamma \gamma \rightarrow \pi \pi$ are described, both for the total cross section and for the angular distributions simultaneously for $\gamma \gamma \rightarrow \pi^{+} \pi^{-}$and $\gamma \gamma \rightarrow \pi^{0} \pi^{0}$ at all experimentally available energies.
\end{abstract}

The European Physical Society Conference on High Energy Physics -EPS-HEP2013

18-24 July 2013

Stockholm, Sweden

\footnotetext{
* Speaker.

${ }^{\dagger}$ This work is supported by the Polish grant N N202 236640.
} 


\section{Introduction}

Ultrarelativistic colliding heavy ions are a source of high-energy $\gamma \gamma$ collisions. Recently we have studied several processes initiated by the photon-photon collisions such as $\rho^{0} \rho^{0}[1], \mu^{+} \mu^{-}$ [2], $Q \bar{Q}[3], J / \Psi J / \Psi$ [4] and $\pi \pi[5,6]$. We have shown there that inclusion of realistic charge form factors, being Fourier transforms of realistic charge distributions, is crucial to estimate reliable nuclear cross sections. Here we consider $\mathrm{PbPb} \rightarrow \mathrm{PbPb} \pi^{+} \pi^{-}$and $\mathrm{PbPb} \rightarrow \mathrm{PbPb} \pi^{0} \pi^{0}$ reactions. To calculate nuclear cross section, we have to take into account the correct form of the elementary cross section for $\gamma \gamma \rightarrow \pi \pi$ reactions at low, intermediate and high sub-energies.

\section{Elementary cross section}

The $\gamma \gamma \rightarrow \pi \pi$ reaction is rather complicated. We have tried to understand both $\gamma \gamma \rightarrow \pi^{+} \pi^{-}$ and $\gamma \gamma \rightarrow \pi^{0} \pi^{0}$ processes simultaneously, starting from the two-pion threshold ( $\mathrm{W}=2 m_{\pi}$ ) up to the maximal experimentally available energy $W_{\gamma \gamma} \approx 6 \mathrm{GeV}$. In Fig. 1 we show our model results (left and middle panel) against world data for $\gamma \gamma \rightarrow \pi \pi$. We get a good agreement with all available data for the first time in such a large range of energies. In the right side of Fig. 1 we present a ratio of the neutral and charged pion pair cross sections as a function of $\gamma \gamma$ energy. The upper line represents the handbag model result, which is independent of $z=\cos \theta$ and of the collision energy. Our result, which includes the Brodsky-Lepage pQCD [7] and the handbag mechanism proposed by Diehl, Kroll and Vogt [8]), describes the experimental data measured by the Belle Collaboration.
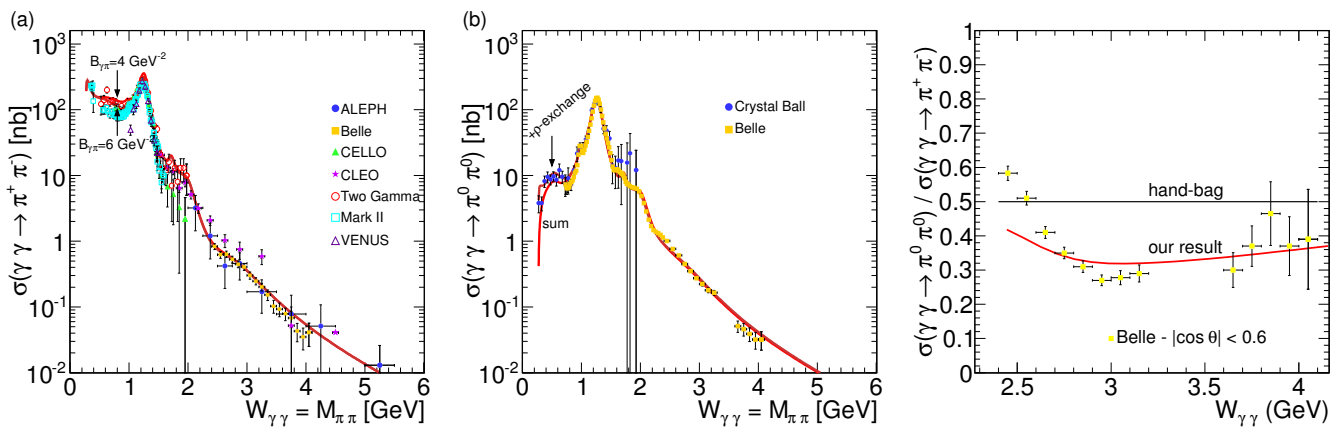

Figure 1: Results of our fit: $\gamma \gamma \rightarrow \pi^{+} \pi^{-}(|\cos \theta|<0.6)$ (left panel) and $\gamma \gamma \rightarrow \pi^{0} \pi^{0}(|\cos \theta|<0.8)$ (middle panel). The right panel: the ratio of the $\gamma \gamma \rightarrow \pi^{0} \pi^{0}$ and $\gamma \gamma \rightarrow \pi^{+} \pi^{-}$cross sections as a function of $\sqrt{{ }^{s \gamma \gamma}}$.

\section{Nuclear cross section}

The nuclear cross section has been calculated with the help of $b$-space equivalent photon approximation (EPA). This approach allows to separate ultraperipheral collisions of nuclei $(b>$ $R_{1}+R_{2} \approx 14 \mathrm{fm}$ ). A compact formula for calculating the total cross section takes the form:

$$
\sigma\left(A A \rightarrow A A \pi \pi ; s_{A A}\right)=\int \hat{\sigma}\left(\gamma \gamma \rightarrow \pi \pi ; W_{\gamma \gamma}\right) S_{a b s}^{2}(\mathbf{b}) N\left(\omega_{1}, \mathbf{b}_{1}\right) N\left(\omega_{2}, \mathbf{b}_{2}\right) \frac{W_{\gamma \gamma}}{2} d^{2} \mathbf{b}_{1} d^{2} \mathbf{b}_{2} d W_{\gamma \gamma} d Y_{\pi \pi} .
$$

The details of its derivation can be found in our papers $[2,6]$. 
Fig. 2 shows two-dimensional distributions in pseudorapidity of charged (left panel) or neutral pion (right panel) and transverse momentum of one of the pions. With larger $p_{t, \pi}$ values, the pseudorapidity distribution becomes somewhat narrower.
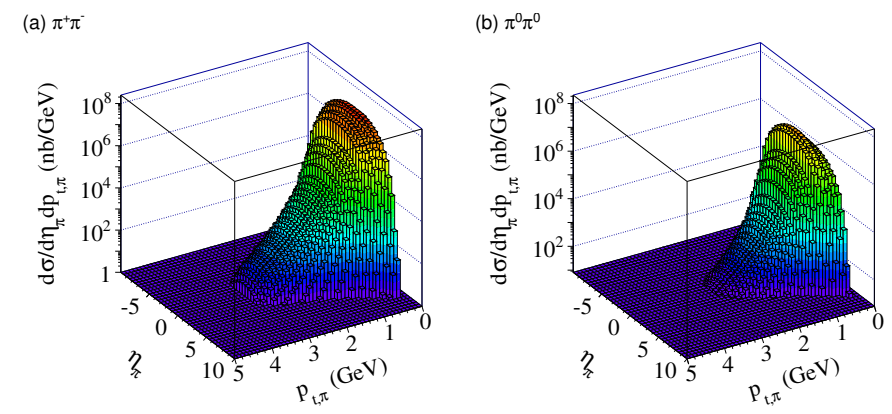

Figure 2: $\frac{d \sigma}{d \eta_{\pi} d p_{t, \pi}}$ for the ${ }^{208} \mathrm{~Pb}^{208} \mathrm{~Pb} \rightarrow{ }^{208} \mathrm{~Pb}^{208} \mathrm{~Pb} \pi^{+} \pi^{-}$reaction (left panel) and for the ${ }^{208} \mathrm{~Pb}^{208} \mathrm{~Pb} \rightarrow{ }^{208} \mathrm{~Pb}^{208} \mathrm{~Pb} \pi^{0} \pi^{0}$ reaction (right panel) at the LHC energy.

\section{Conclusions}

We have shown that in order to describe the word data for $\gamma \gamma \rightarrow \pi^{+} \pi^{-}$and $\gamma \gamma \rightarrow \pi^{0} \pi^{0}$ reactions, one should include several different mechanisms: soft two-pion continuum, several resonanses, pion-pion rescattering, pQCD mechanisms proposed by Brodsky and Lepage, as well as the hand-bag mechanism proposed by Diehl, Kroll and Vogt. The energy-dependent cross sections for these two sub-processes have been used next in EPA in the impact parameter space to calculate for the first time corresponding production rate in ultraperipheral ultrarelativistic heavy-ion reactions. In this calculation we have taken into account realistic charge distributions in colliding nuclei.

The $\gamma \gamma \rightarrow \pi^{+} \pi^{-}$sub-process constitutes a background to the exclusive $A A \rightarrow A \rho^{0}\left(\rightarrow \pi^{+} \pi^{-}\right) A$ process, initiated by photon-pomeron or pomeron-photon sub-processes. We have found that only a part of the dipion invariant mass spectrum associated with $\gamma \gamma$-collisions can be potentially visible as the cross section for the $A A \rightarrow A \rho^{0} A$ reaction is very large [9].

\section{References}

[1] M. Kłusek, A. Szczurek and W. Schäfer, Phys. Lett. B674 92 (2009).

[2] M. Kłusek-Gawenda and A. Szczurek, Phys. Rev. C82 014904 (2010).

[3] M. Kłusek-Gawenda, A. Szczurek, M.V.T. Machado and V.G. Serbo, Phys. Rev. C83 024903 (2011).

[4] S. Baranov, A. Cisek, M. Kłusek-Gawenda, W. Schäfer and A. Szczurek, Eur. Phys. J. C73 2335 (2013).

[5] M. Kłusek-Gawenda and A. Szczurek, Phys. Lett. B700 322 (2011).

[6] M. Kłusek-Gawenda and A. Szczurek, Phys. Rev. C87 054908 (2013).

[7] S.J. Brodsky and G.P. Lepage, Phys. Rev. D24 1808 (1981).

[8] M. Diehl, P. Kroll and C. Vogt, Phys. Lett. $B 53299$ (2002); M. Diehl and P. Kroll, Phys. Lett. B683 165 (2010).

[9] M. Kłusek-Gawenda and A. Szczurek, arXiv: 1309.2463 [nucl-th]. 